\title{
THE EFFECT OF AN ASYMMETRIC SUBMARINE TRENCH ON THE EFFICIENCY OF AN OSCILLATING WATER COLUMN DEVICE IN A TWO-LAYER FLUID
}

\author{
AYRTON ALFONSO MEDINA-RODRÍGUEZ, ALEJANDRO MARTÍNEZ \& RODOLFO SILVA CASARÍN \\ Instituto de Ingeniería, UNAM Cd Universitaria, México
}

\begin{abstract}
The effect of a submarine asymmetric trench on the efficiency of an Oscillating Water Column (OWC) device in a two-layer fluid is analyzed within the context of linearized water wave theory. Under the potential flow approach, the associated boundary value problem is solved by the matched eigenfunction expansion method. Numerical results for the OWC device efficiency for several physical parameters and configurations were obtained. Three different positions of the submarine trench were considered. The effects of the submarine trench depths and the distance of the trench from the surface piercing barrier on the efficiency of the OWC device are discussed in detail. In addition to the structural properties, the OWC performance is dependent on the fluid density ratio and the interface location. In order to verify the computational results, these are compared with results published in specialized literature and very good agreement was achieved.

Keywords: oscillating water column, submarine trench, linearized wave theory, two-layer fluid, efficiency of oscillating water column.
\end{abstract}

\section{INTRODUCTION}

The study of the efficiency of an Oscillating Water Column device in a single fluid domain of homogeneous density has been extensively investigated in recent years and remains a topic of great interest in linear wave theory. In this context, several relevant analytical, numerical and experimental investigations have been performed. However, there has been negligible progress in analyzing the performance of a OWC device in a two-layer or stratified fluid with different but constant densities. Density stratification, which is due to solar heating or the mixing of fresh water with saline water, is a common phenomenon in the ocean and is of increasing interest, since the physical consequences are significant in a wide range of applications, particularly marine science and ocean renewable energy.

In a two-layer fluid, the fluid in each layer is assumed to be inviscid, incompressible and have a constant density, with the upper, lighter fluid, of density $\rho_{1}$ lying over the heavier fluid of density $\rho_{2}$ and there are two wave modes types because there is a free surface and an interface [1]. Among the various aspects of this problem, a major concern is the transformation of wave energy from surface to internal waves and vice versa, Wehausen and Laitone [2]. As a result, for each mode, the wave elevations for the free surface and for the interface must be analyzed, due to the changing bathymetry of the ocean floor.

The objective of this work is to analyze the efficiency of an OWC device associated with the transformation of energy between surface and internal waves and vice versa, due to the presence of a nearby trench. To the authors' knowledge, to date, no studies have been made on an OWC device in a two-layer fluid where the immersed tip of the front barrier is located both above and below the interface.

Using the eigenfunction expansion method, a system of equations are obtained for the determination of the unknowns in surface and internal wave modes. Numerical estimates for the efficiency of the OWC device are then obtained for several physical parameters involved in the problem. In order to verify the computational results, numerical results for particular cases are compared with results obtained earlier by Evans and Porter [3] and Rezanejad et al. [4]. 


\section{THE BOUNDARY-VALUE PROBLEM}

In the present study, the Cartesian coordinate system was chosen, with the $x$ axis corresponding to the opposite direction of the wave propagation and the $z$ axis corresponding to the upward direction. The origin of the coordinate system lies on the undisturbed water surface and the rigid, vertical wall. The OWC is integrated by a rigid wall, situated at $x=0$, extending down to the bottom and complemented by a thin, vertical, surface-piercing barrier, at $x=b$, with a draft, $h_{a}$, as shown in Fig. 1 . A turbine is connected to the air chamber formed by the two walls. The distance of the submarine trench from the wall located at $x=b$, is $L$ and the width of the trench is equal to $L_{2}$.

In the two-layer fluid, the upper fluid has an undisturbed free surface located at $z=0$ and the two fluids are separated by a common undisturbed interface located at $z=-h$. The upper and lower fluid domains are $0<x<\infty$ (see Fig. 1). The domains are divided into four regions, region $1\left(S_{2}<x<\infty,-h_{1}<z\right)$, region $2(S<$ $\left.x<S_{2},-h_{3}<z\right)$, region $3\left(b<x<S,-h_{2}<z\right)$ and region $4\left(0<x<b,-h_{2}<z\right)$, with $S=b+L$ and $S_{2}=S+L_{2}$. The rigid wall is denoted by $L_{b}=\{(x, z): x=$ $\left.S,-h_{a} \leq z \leq 0\right\}$, the gap between the immersed tip of the barrier and the bottom by $L_{g}=\left\{(x, z):-h_{2} \leq z \leq-h_{a}\right\}$, the rigid vertical wall by $S_{w}=\left\{(x, z): x=0,-h_{2}<\right.$ $z<0\}$, the internal free surface inside the water column by $S_{i}=\{(x, z): 0 \leq x \leq$ $b, z=0\}$, the external free surface by $S_{f}=\{(x, z): b \leq x \leq \infty, z=0\}$ and the bottom by $S_{b}=\left\{(x, z):\left(0<x<S, z=-h_{2}\right) \cup\left(x=S,-h_{3}<z<-h_{2}\right) \cup\left(S<x<S_{2}, z=\right.\right.$ $\left.\left.-h_{3}\right) \cup\left(x=S_{2},-h_{3}<z<-h_{1}\right) \cup\left(S_{2}<x<\infty, z=-h_{1}\right)\right\}$.

The fluid is assumed to be inviscid and incompressible and linear wave theory is applied, ignoring the effect of surface tension. An irrotational flow and a simple harmonic in time with angular frequency $\omega$ are assumed. There is thus a velocity potential $\Phi_{j}(x, z, t)$ with $\Phi(x, z, t)=\operatorname{Re}\left\{\phi_{j}(x, z) \mathrm{e}^{-i \omega t}\right\}$ for $j=1,2,3$ and 4 , where $\operatorname{Re}\{\}$ denotes the real part of a complex expression and $t$ is the time. The spatial velocity potential $\phi_{j}$ for $j=1,2,3$ and 4

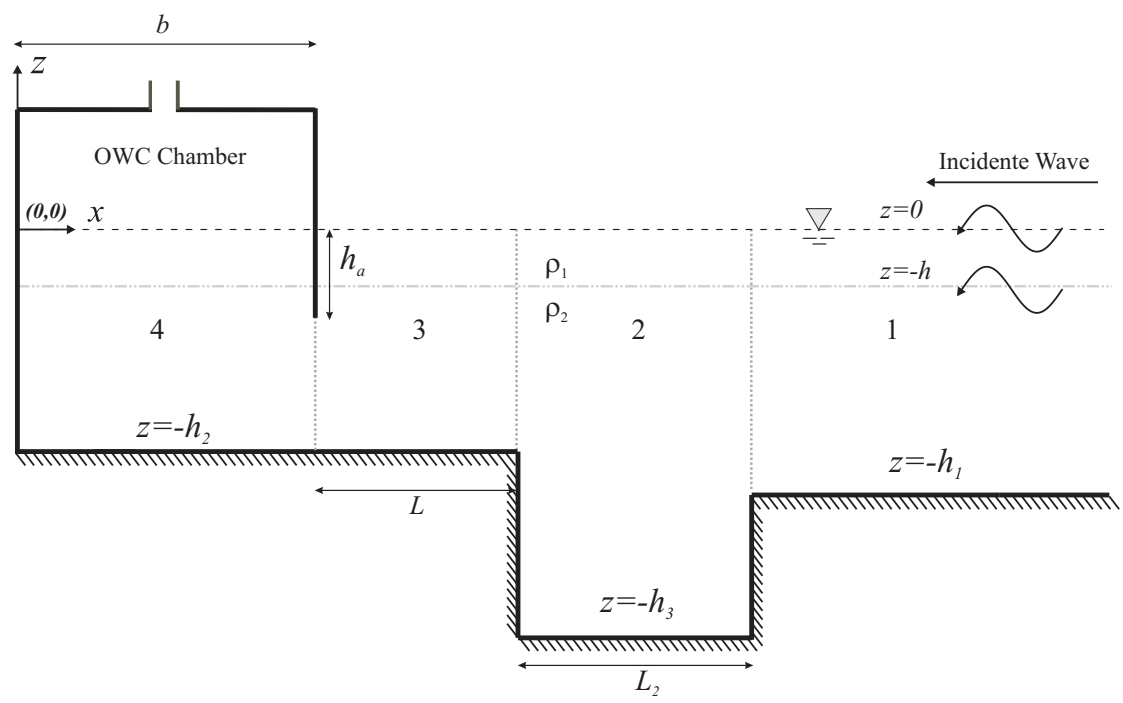

Figure 1: Definition sketch of an OWC device near an asymmetric submarine trench in a two-layer fluid. 
satisfies the Laplace equation:

$$
\frac{\partial^{2} \phi_{j}}{\partial x^{2}}+\frac{\partial^{2} \phi_{j}}{\partial z^{2}}=0
$$

In addition, $\phi$ satisfies the no-flow condition on the solid boundaries given by:

$$
\begin{gathered}
\frac{\partial \phi}{\partial z}=0 \text { on }\left\{\begin{array}{lll}
z=-h_{1} & \text { for } & S_{2}<x<\infty, \\
z=-h_{2} & \text { for } & 0<x<S, \\
z=-h_{3} & \text { for } & S<x<S_{2},
\end{array}\right.
\end{gathered}
$$

The boundary condition on the rigid vertical wall is described as:

$$
\frac{\partial \phi}{\partial x}=0 \quad \text { on } \quad x=0, \quad-h_{2}<z<0 .
$$

The boundary condition on the barrier is given by:

$$
\frac{\partial \phi}{\partial x}=0 \quad \text { on } \quad x=b, \quad-h_{a}<z<0 .
$$

Since it is assumed that the motion is harmonic in time, an imposed pressure distribution over the internal free surface is of the form $P(t)=\operatorname{Re}\left\{p e^{-i \omega t}\right\}$; thus, the linearized free surface boundary condition is given by:

$$
\begin{gathered}
\frac{\partial \phi}{\partial z}-K \phi=0 \quad \text { on } \quad z=0, \quad b<x<\infty, \\
\frac{\partial \phi}{\partial z}-K \phi=\frac{i \omega p}{\rho g} \quad \text { on } \quad z=0, \quad 0<x<b,
\end{gathered}
$$

where $K=\omega^{2} / g$ and $g$ is the gravitational constant. Assuming that the pressure is continuous at the interface, the linearized dynamic and kinematic conditions at the mean interface yield this equation (see Wehausen and Laitone [2]):

$$
\begin{gathered}
\left(\frac{\partial \phi_{j}}{\partial z}\right)_{z=-h_{+}}=\left(\frac{\partial \phi_{j}}{\partial z}\right)_{z=-h_{-}}, \\
s\left(\frac{\partial \phi_{j}}{\partial z}-K \phi_{j}\right)_{z=-h_{+}}=\left(\frac{\partial \phi_{j}}{\partial z}-K \phi_{j}\right)_{z=-h_{-}},
\end{gathered}
$$

with $j=1,2,3$ and 4 , and $s=\rho_{1} / \rho_{2}$ with $0<s<1$.

The continuity of pressure and horizontal velocity across the vertical planes separating the fluid regions are given by (Kirby and Dalrymple [5] and Rezanejad et al. [4]):

$$
\begin{aligned}
\phi_{1}=\phi_{2}, & \frac{\partial \phi_{1}}{\partial x}=\frac{\partial \phi_{2}}{\partial x}, \quad \text { on } \quad x=S_{2}, \quad-h_{1} \leq z \leq 0, \\
\phi_{2}=\phi_{3}, & \frac{\partial \phi_{2}}{\partial x}=\frac{\partial \phi_{3}}{\partial x}, \quad \text { on } \quad x=S, \quad-h_{2} \leq z \leq 0,
\end{aligned}
$$




$$
\phi_{3}=\phi_{4}, \quad \frac{\partial \phi_{3}}{\partial x}=\frac{\partial \phi_{4}}{\partial x}, \quad \text { on } \quad x=b, \quad-h_{2} \leq z \leq-h_{a} .
$$

Following the method of Evans and Porter [3] the total potential $\phi(x, z)$ is decomposed into two parts as follows:

$$
\phi(x, z)=\phi^{S}+\frac{i \omega p}{\rho g} \phi^{R} .
$$

The scattered potential $\phi^{S}$ satisfies eqns (1)-(5) and (7)-(11) with $p=0$, while $\phi^{R}$ satisfies eqns (1)-(4) and (6)-(11) with eqn (6) replaced by:

$$
\frac{\partial \phi^{R}}{\partial z}-K \phi^{R}=1 \quad \text { on } \quad z=0, \quad 0<x<b .
$$

In addition, the far-field behavior for the scattering and radiation problem are of the form:

$$
\phi^{S}(x, z) \sim \sum_{j=I}^{I I}\left(\mathrm{e}^{-i k_{j}\left(x-S_{2}\right)}+R_{j} \mathrm{e}^{i k_{j}\left(x-S_{2}\right)}\right) \psi_{j}\left(k_{j}, z\right), \quad \text { as } \quad x \rightarrow+\infty
$$

and

$$
\phi^{R}(x, z) \sim \sum_{j=I}^{I I} A_{j}^{R} \mathrm{e}^{-i k_{j}\left(x-S_{2}\right)} \psi_{j}\left(k_{j}, z\right), \quad \text { as } \quad x \rightarrow+\infty,
$$

respectively, where $A_{j}^{R}$ with $j=I, I I$, is related to the amplitude of the surface and internal waves radiated to $+\infty$, due to the forcing on the internal free surface; $R_{I}$ and $R_{I I}$ are the unknown constants associated with the reflection of incident surface and internal waves in the scattering problem of the waves of wave numbers $k_{I}$ and $k_{I I}$ in region 1, respectively; $\psi_{j}\left(k_{j}, z\right)$ is the associated vertical eigenfunction given by:

$$
\psi_{j}= \begin{cases}\frac{N_{j}^{-1} \sinh k_{j}\left(h_{1}-h\right)\left(k_{j} \cosh k_{j} z+K \sinh k_{j} z\right)}{K \cosh k_{j} h-k_{j} \sinh k_{j} h} & \text { for } \quad-h<z<0, \\ N_{j}^{-1} \cosh k_{j}\left(z+h_{1}\right) & \text { for } \quad-h_{1}<z<-h,\end{cases}
$$

where

$$
\begin{gathered}
N_{j}^{2}=\left(4 k_{j}\right)^{-1}\left(K \cosh k_{j} h-k_{j} \sinh k_{j} h\right)^{-2} \\
{\left[\left(K \cosh k_{j} h-k_{j} \sinh k_{j} h\right)^{2}\left[2\left(h_{1}-h\right) k_{j}+\sinh 2 k_{j}\left(h_{1}-h\right)\right]-\right.} \\
s \sinh ^{2} k_{j}\left(h_{1}-h\right)\left[K^{2}\left(2 k_{j} h-\sinh 2 k_{j} h\right)-\right. \\
\left.\left.k_{j}^{2}\left(2 k_{j} h+\sinh 2 k_{j} h\right)+2 K k_{j}\left(\cosh 2 k_{j} h-1\right)\right]\right],
\end{gathered}
$$

with $j=I$ and $I I$. In addition, $k_{j}$ are the roots of the dispersion relation in surface and internal modes, where wave numbers $k_{I}$ and $k_{I I}$ indicate the positive real roots and $\mathrm{i} k_{n}$ for $n \geq 1$ indicate the purely imaginary roots of the dispersion relation given by:

$$
\begin{aligned}
&(1-s) k^{2} \tanh k\left(h_{1}-h\right) \tanh k h-k K[\left.\tanh k h+\tanh k\left(h_{1}-h\right)\right]+ \\
& K^{2}\left[s \tanh k\left(h_{1}-h\right) \tanh k h+1\right]=0 .
\end{aligned}
$$


In addition to $R_{j}$ and $A_{j}^{R}$, of considerable interest is the induced volume flux across the internal free surface, $Q(t)=\operatorname{Re}\left\{q e^{-i \omega t}\right\}$ so that (Evans and Porter [3]):

$$
q=\int_{S_{i}} \frac{\partial \phi}{\partial z} d x=q^{S}+\frac{i \omega p}{\rho g} q^{R},
$$

where $q^{S}$ and $q^{R}$ are the volume fluxes across $S_{i}$ in the scattering and radiation problems, respectively. Using the continuity of volume flux across the internal free surface and the gap between the barrier tip and the bottom, we obtain:

$$
q^{S, R}=\int_{S_{i}} \frac{\partial \phi}{\partial z} d x=\int_{L_{g}} \frac{\partial \phi}{\partial x} d z .
$$

The volume flux $q^{R}$ for the radiation problem is separated into real and imaginary parts as in [3]:

$$
\frac{i \omega p}{\rho g} q^{R}=(\tilde{B}-i \tilde{A}) p
$$

where $\tilde{A}$ and $\tilde{B}$ are analogous to the added mass and the radiation damping coefficients in a rigid body system and following Evans and Porter [3], are called the radiation susceptance and the radiation conductance parameters and are given as follows:

$$
\begin{aligned}
& \tilde{A}=\frac{\omega}{\rho g} \operatorname{Re}\left\{q^{R}\right\}, \\
& \tilde{B}=\frac{\omega}{\rho g} \operatorname{Re}\left\{q^{R}\right\} .
\end{aligned}
$$

Spatial potential $\phi^{R}$ and the complex conjugate $\bar{\phi}^{R}$ both satisfy the governing eqn (1) and the same boundary conditions. We use Green's integral theorem on $\phi^{R}$ and $\bar{\phi}^{R}$, given by:

$$
\int_{C}\left(\phi^{R} \frac{\partial \bar{\phi}^{R}}{\partial n}-\bar{\phi}^{R} \frac{\partial \phi^{R}}{\partial n}\right) d s=0,
$$

where the closed boundary $C$ is composed of $C=S_{b}+S_{w}+S_{i}+S_{f}+L_{b}+S_{\infty}$, where $S_{\infty}$ is the fictitious far boundary as $x \rightarrow \infty$. Due to the free surface condition and the rigid structural boundary conditions, the lines $S_{f}, S_{b}, S_{w}$ and $L_{b}$ have no contributions.

In addition, using the condition at the interface and the result given by (Das et al. [6]; Panda and Martha [7]):

$$
\int_{-h_{1}}^{-h} \psi_{I}^{-} \psi_{I I}^{-} d z+s \int_{-h}^{0} \psi_{I}^{+} \psi_{I I}^{+} d z=0
$$

where superscripts ${ }^{+}$and - indicate the upper and lower layer vertical eigenfunctions, respectively, and after taking the contribution from the lines $S_{\infty}$ and $S_{i}$ and eqns (15) and (19), an explicit expression for $\tilde{B}$, in terms of the complex amplitude of the radiated surface and internal waves, $A_{I}^{R}$ and $A_{I I}^{R}$, respectively, is obtained in the form:

$$
\tilde{B}=\frac{\omega}{\rho g} K\left(k_{I}\left|A_{I}^{R}\right|^{2}+k_{I I}\left|A_{I I}^{R}\right|^{2}\right) .
$$

In addition, based on the assumption that there is a linear relation between the volume flux through the turbine and the pressure drop across it, an expression for the efficiency of the 
device is derived. Therefore, we assume:

$$
q=\Lambda p
$$

where $\Lambda$ is a real control parameter, so that the following expression for the maximum efficiency is obtained:

$$
\eta_{\max }=\frac{2 \tilde{B}}{\Lambda_{o p t}+\tilde{B}}
$$

where

$$
\Lambda_{\text {opt }}=\left(\tilde{B}^{2}+\tilde{A}^{2}\right)^{\frac{1}{2}}
$$

Details of the derivation of such equations can be found in Evans [8] or Smith [9].

\section{SOLUTION}

The matched eigenfunction expansion method described by Rezanejad et al. [4], for the case of a single chamber on a stepped bottom, was extended to obtain the velocity potentials for a single chamber in a two-layer fluid close to a submarine trench. Hence, the details are omitted here to avoid repetition. The spatial velocity potentials in the four regions are expanded in terms of the appropriate eigenfunctions as given by:

$$
\begin{gathered}
\phi_{1}^{R}(x, z)=\sum_{j=I}^{I I} A_{j}^{R} e^{i k_{j}\left(x-S_{2}\right)} \psi_{j}+\sum_{n=1}^{\infty} A_{n}^{R} e^{-k_{n}\left(x-S_{2}\right)} \psi_{n}, \\
\phi_{2}^{R}(x, z)=\sum_{j=I}^{I I}\left(E_{j}^{R} \frac{\cos w_{j}\left(S_{2}-x\right)}{\cos w_{j} L_{2}}+F_{j}^{R} \frac{\sin w_{j}\left(S_{2}-x\right)}{\sin w_{j} L_{2}}\right) G_{j}+ \\
\sum_{n=1}^{\infty}\left(E_{n}^{R} \frac{\cosh w_{n}\left(S_{2}-x\right)}{\cosh w_{n} L_{2}}+F_{n}^{R} \frac{\sinh w_{n}\left(S_{2}-x\right)}{\sinh w_{n} L_{2}}\right) G_{n}, \\
\phi_{3}^{R}(x, z)=\sum_{j=I}^{I I}\left(C_{j}^{R} \frac{\cos p_{j}(S-x)}{\cos p_{j} L}+D_{j}^{R} \frac{\sin p_{j}(S-x)}{\sin p_{j} L}\right) M_{j}+ \\
\sum_{n=1}^{\infty}\left(C_{n}^{R} \frac{\cosh p_{n}(S-x)}{\cosh p_{n} L}+D_{n}^{R} \frac{\sinh p_{n}(S-x)}{\sinh p_{n} L}\right) M_{n}, \\
\phi_{4}^{R}(x, z)=\sum_{j=I}^{I I} B_{j}^{R} \cos p_{j} x M_{j}+\sum_{n=1}^{\infty} B_{n}^{R} \cosh p_{n} x M_{n}+\frac{1}{K},
\end{gathered}
$$

with the eigenfunctions $M_{j}$ and $G_{j}$ as given by:

$$
M_{j}= \begin{cases}\frac{L_{j}^{-1} \sinh p_{j}\left(h_{2}-h\right)\left(p_{j} \cosh p_{j} z+K \sinh p_{j} z\right)}{K \cosh p_{j} h-p_{j} \sinh p_{j} h} & \text { for } \quad-h<z<0, \\ L_{j}^{-1} \cosh p_{j}\left(z+h_{2}\right) & \text { for } \quad-h_{2}<z<-h,\end{cases}
$$




$$
G_{j}= \begin{cases}\frac{Y_{j}^{-1} \sinh w_{j}\left(h_{3}-h\right)\left(w_{j} \cosh w_{j} z+K \sinh w_{j} z\right)}{K \cosh w_{j} h-w_{j} \sinh w_{j} h} & \text { for } \quad-h<z<0, \\ Y_{j}^{-1} \cosh w_{j}\left(z+h_{3}\right) & \text { for } \quad-h_{3}<z<-h,\end{cases}
$$

while the terms $L_{j}$ and $W_{j}$ for $j=I, I I$ are given as follows:

$$
\begin{gathered}
L_{j}^{2}=\left(4 p_{j}\right)^{-1}\left(K \cosh p_{j} h-p_{j} \sinh p_{j} h\right)^{-2} \\
{\left[\left(K \cosh p_{j} h-p_{j} \sinh p_{j} h\right)^{2}\left[2\left(h_{2}-h\right) p_{j}+\sinh 2 p_{j}\left(h_{2}-h\right)\right]-\right.} \\
s \sinh ^{2} p_{j}\left(h_{2}-h\right)\left[K^{2}\left(2 p_{j} h-\sinh 2 p_{j} h\right)-\right. \\
\left.\left.p_{j}^{2}\left(2 p_{j} h+\sinh 2 p_{j} h\right)+2 K p_{j}\left(\cosh 2 p_{j} h-1\right)\right]\right], \\
Y_{j}^{2}=\left(4 w_{j}\right)^{-1}\left(K \cosh w_{j} h-w_{j} \sinh w_{j} h\right)^{-2} \\
{\left[\left(K \cosh w_{j} h-w_{j} \sinh w_{j} h\right)^{2}\left[2\left(h_{3}-h\right) w_{j}+\sinh 2 w_{j}\left(h_{3}-h\right)\right]-\right.} \\
s \sinh ^{2} w_{j}\left(h_{3}-h\right)\left[K^{2}\left(2 w_{j} h-\sinh 2 w_{j} h\right)-\right. \\
\left.\left.w_{j}^{2}\left(2 w_{j} h+\sinh 2 w_{j} h\right)+2 K w_{j}\left(\cosh 2 w_{j} h-1\right)\right]\right] .
\end{gathered}
$$

On the other hand, $M_{n}, G_{n}, L_{n}$ and $Y_{n}$ for $n \geq 1$ are defined by eqns (28)-(35) with the substitution of $p_{j}$ and $w_{j}$ by $i p_{n}$ and $i w_{n}$, respectively.

The positive real roots $p_{I}$ and $p_{I I}$, and the purely imaginary roots $i p_{n}$ for $n \geq 1$ of the dispersion relation in $p$ are given by

$$
\begin{aligned}
& (1-s) p^{2} \tanh p\left(h_{2}-h\right) \tanh p h-p K\left[\tanh p h+\tanh p\left(h_{2}-h\right)\right]+ \\
& K^{2}\left[s \tanh p\left(h_{2}-h\right) \tanh p h+1\right]=0 .
\end{aligned}
$$

The wave numbers $w_{I}$ and $w_{I I}$ are positive real roots and $i w_{n}$ for $n \geq 1$ are purely imaginary roots of the dispersion relation

$$
\begin{aligned}
(1-s) w^{2} \tanh w\left(h_{3}-h\right) \tanh w h-w K\left[\tanh w h+\tanh w\left(h_{3}-h\right)\right]+ \\
K^{2}\left[s \tanh w\left(h_{3}-h\right) \tanh w h+1\right]=0,
\end{aligned}
$$

where $0<k_{I}<k_{I I}, k_{n}>0 ; 0<p_{I}<p_{I I}, p_{n}>0 ; 0<w_{I}<w_{I I}, w_{n}>0$.

The eigenfunctions $\psi_{n}$ 's, $M_{n}$ 's and $G_{n}$ 's with $n=I, I I, 1,2, \ldots$ are orthonormal with respect to the inner product

$$
\begin{aligned}
\left\langle\psi_{n}, \psi_{m}\right\rangle & =\int_{-h_{1}}^{-h} \psi_{n}(z) \psi_{m}(z) d z+s \int_{-h}^{0} \psi_{n}(z) \psi_{m}(z) d z=\delta_{n m}, \\
\left\langle M_{n}, M_{m}\right\rangle & =\int_{-h_{2}}^{-h} M_{n}(z) M_{m}(z) d z+s \int_{-h}^{0} M_{n}(z) M_{m}(z) d z=\delta_{n m}, \\
\left\langle G_{n}, G_{m}\right\rangle & =\int_{-h_{3}}^{-h} G_{n}(z) G_{m}(z) d z+s \int_{-h}^{0} G_{n}(z) G_{m}(z) d z=\delta_{n m},
\end{aligned}
$$

where $\delta_{n m}$ is the Kronecker delta. 
The coefficients $A_{n}^{R}, B_{n}^{R}, C_{n}^{R}, D_{n}^{R}, E_{n}^{R}$ and $F_{n}^{R}$, with $n=I, I I, 1,2, \ldots$ in eqns (29)(32), are the unknown constants to be determined. Application of the matching conditions as in eqns (9) and (11) on the velocity potentials as described by eqns (29)-(32), exploiting the orthonormality of the eigenfunctions as defined in eqns (38)-(40) and truncating the infinite series after $\mathrm{N}$ terms, gives the following system of equations for the determination of the unknowns:

$$
\begin{gathered}
\sum_{j=I}^{I I} i k_{j} A_{j}^{R} \int_{-h_{1}}^{0} \psi_{j} P_{m} d z+\sum_{j=I}^{I I}\left(\frac{w_{j}}{\sin w_{j} L_{2}}\right) F_{j}^{R} \delta_{j m}-\sum_{n=1}^{\infty} k_{n} A_{n}^{R} \int_{-h_{1}}^{0} \psi_{n} P_{m} d z+ \\
\sum_{n=1}^{\infty}\left(\frac{w_{n}}{\sinh w_{n} L_{2}}\right) F_{n}^{R} \delta_{n m}=0 \quad \text { for } \quad m=I, I I, 1,2, \ldots, \\
\sum_{j=I}^{I I} A_{j}^{R} \delta_{j m}-\sum_{j=I}^{I I}\left(\frac{1}{\cos w_{j} L_{2}}\right) E_{j}^{R} \int_{-h_{1}}^{0} G_{j} \psi_{m} d z+\sum_{j=I}^{I I} A_{n}^{R} \delta_{n m}- \\
\sum_{n=1}^{\infty}\left(\frac{1}{\cosh w_{n} L_{2}}\right) E_{n}^{R} \int_{-h_{1}}^{0} G_{n} \psi_{m} d z=0 \quad \text { for } \quad m=I, I I, 1,2, \ldots,
\end{gathered}
$$

$$
\begin{aligned}
& \sum_{j=I}^{I I}\left(\frac{p_{j}}{\sin p_{j} L}\right) D_{j}^{R} \int_{-h_{2}}^{0} M_{j} G_{m} d z- \\
& \sum_{j=I}^{I I} w_{j}\left(-E_{j}^{R} \tan w_{j} L_{2}+F_{j}^{R} \cot w_{j} L_{2}\right) \delta_{j m}+\sum_{n=1}^{\infty}\left(\frac{p_{n}}{\sinh p_{n} L}\right) D_{n}^{R} \int_{-h_{2}}^{0} M_{n} G_{m} d z- \\
& \sum_{n=1}^{\infty} w_{n}\left(E_{n}^{R} \tanh w_{n} L_{2}+F_{n}^{R} \operatorname{coth} w_{n} L_{2}\right) \delta_{n m}=0 \quad \text { for } \quad m=I, I I, 1,2, \ldots,
\end{aligned}
$$

$$
\begin{gathered}
-\sum_{j=I}^{I I}\left(\frac{1}{\cos p_{j} L}\right) C_{j}^{R} \delta_{j m}+\sum_{j=I}^{I I}\left(E_{j}^{R}+F_{j}^{R}\right) \int_{-h_{2}}^{0} G_{j} M_{m} d z- \\
\sum_{n=1}^{\infty}\left(\frac{1}{\cosh p_{n} L}\right) C_{n}^{R} \delta_{n m}+\sum_{n=1}^{\infty}\left(E_{n}^{R}+F_{n}^{R}\right) \int_{-h_{2}}^{0} G_{n} M_{m} d z=0 \\
\text { for } \quad m=I, I I, 1,2, \ldots,
\end{gathered}
$$

$$
\begin{aligned}
& -\sum_{j=I}^{I I} p_{j} B_{j}^{R} \sin p_{j} b \delta_{j m}+\sum_{j=I}^{I I} p_{j}\left(-C_{j}^{R} \tan p_{j} L+D_{j}^{R} \cot p_{j} L\right) \delta_{j m}+ \\
& \sum_{n=1}^{\infty} p_{n} B_{n}^{R} \sinh p_{n} b \delta_{n m}+\sum_{n=1}^{\infty} p_{n}\left(C_{n}^{R} \tanh p_{n} L+D_{n}^{R} \operatorname{coth} p_{n} L\right) \delta_{n m}=0 \\
& \text { for } \quad m=I, I I, 1,2, \ldots,
\end{aligned}
$$




$$
\begin{aligned}
&-\sum_{j=I}^{I I} B_{j}^{R} \cos p_{j} b \int_{-h_{2}}^{-h_{a}} M_{j} M_{m} d z+ \\
& \sum_{j=I}^{I I} C_{j}^{R}\left(\int_{-h_{2}}^{-h_{a}} M_{j} M_{m} d z-p_{j} \tan p_{j} L \int_{-h_{a}}^{0} M_{j} M_{m} d z\right)+ \\
& \sum_{j=I}^{I I} D_{j}^{R}\left(\int_{-h_{2}}^{-h_{a}} M_{j} M_{m} d z+p_{j} \cot p_{j} L \int_{-h_{a}}^{0} M_{j} M_{m} d z\right)+ \\
& \sum_{n=1}^{\infty} C_{n}^{R}\left(\int_{-h_{2}}^{-h_{a}} M_{n} M_{m} d z+p_{n} \tanh p_{n} L \int_{-h_{a}}^{0} M_{n} M_{m} d z\right)+ \\
& \sum_{n=1}^{\infty} D_{n}^{R}\left(\int_{-h_{2}}^{-h_{a}} M_{n} M_{m} d z+p_{n} \operatorname{coth} p_{n} L \int_{-h_{a}}^{0} M_{n} M_{m} d z\right)=\frac{1}{K} M_{-h_{2}}^{-h_{a}} M_{m} d z+
\end{aligned}
$$

\section{RESULTS AND DISCUSSION}

In this section, results based on the analytical method described above are presented. The physical parametric values that were kept constant throughout the numerical computations are $\rho=1025 \mathrm{kgm}^{-3}, g=9.81 \mathrm{~m} \mathrm{~s}^{-2}$. In order to perform the numerical computation, all the infinite systems are truncated up to a finite number of terms $\mathrm{N}$.

Since between 20 and 30 terms are enough to ensure convergence of the numerical results within three decimal places, in the calculations here, all infinite series sums were truncated up to $\mathrm{N}=20$ number of evanescent modes.

In addition, introducing the non-dimensionalised quantities $\mu$ and $\nu$ which represent the radiation susceptance and radiation conductance coefficients, namely:

$$
\mu=\frac{\rho g}{\omega b} \tilde{A}
$$

and

$$
\nu=\frac{\rho g}{\omega b} \tilde{B} .
$$

Therefore, the efficiency $\eta_{\max }$ is given by

$$
\eta_{\max }=\frac{2 \nu}{\left(\nu^{2}+\mu^{2}\right)^{1 / 2}+\nu} .
$$

To validate the method described here, the numerical results for the limiting cases of Evans and Porter [3] and Rezanejad et al. [4] were used. For $s=1$ and $h=0$, the dispersion relations in eqns (18), (36) and (37) are reduced to the dispersion relations in a single layer fluid. In the case of $s=0.97$ and $h / h_{1}=0.05$, the efficiency from this study was compared with the corresponding results of Evans and Porter [3] for an OWC device with a horizontal topography, as shown in Fig. 2(a). The circles in Fig. 2(a) depict the results of Evans and Porter [3], whereas the line represents the results calculated by the present method. It can be seen from Fig. 2(a) that the results are in good agreement. The efficiency obtained for an 


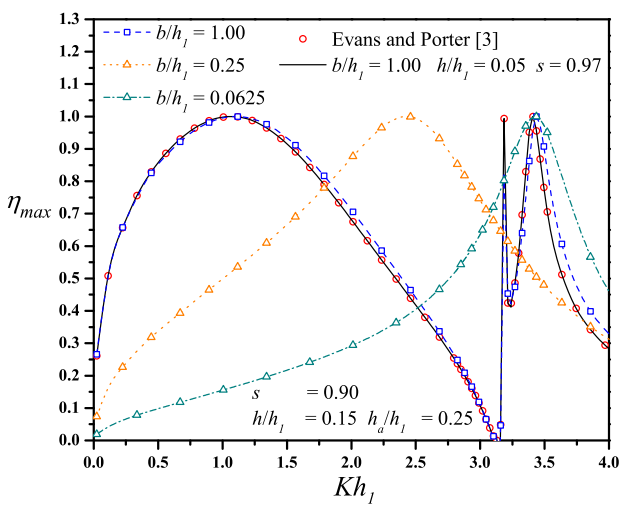

(a)

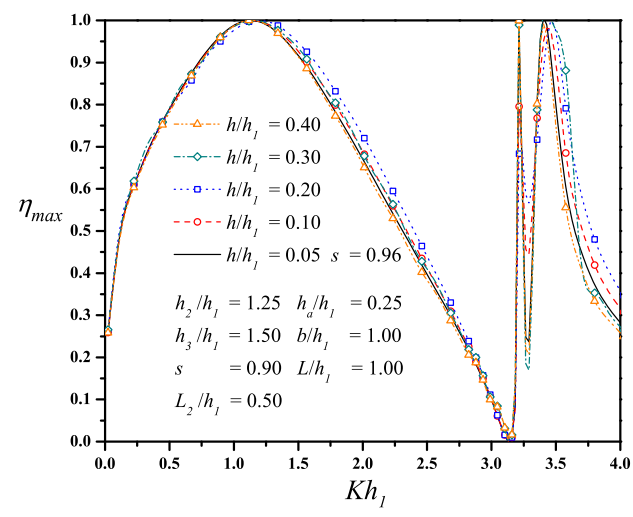

(b)

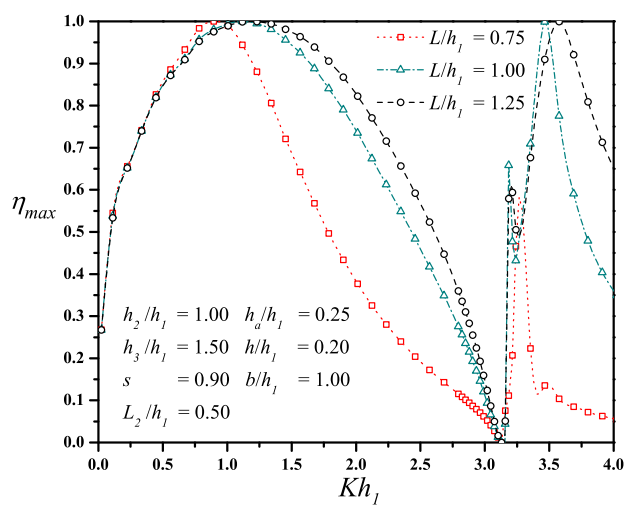

(c)

Figure 2: Analytical results for efficiency versus $K h_{1}$ for various barrier to wall spacings, interface positions and barrier to trench spacings.

OWC device with stepped bottom topography with $s=0.97$ and $h / h_{1}=0.01$, together with the nondimensional parameters $h_{2} / h_{1}=0.5, h_{3} / h_{1}=1.0$ and $h_{a} / h_{1}=b / h_{1}=L / h_{1}=$ $L_{2} / h_{1}=0.25$, was compared with the corresponding results of Rezanejad et al. [4]. The results agree very well, as shown in Fig. 3(a).

Fig. 2(a) shows the results for the efficiency $\eta_{\max }$ versus $K h_{1}$ for various barrier to wall spacings $\left(b / h_{1}=1.0,0.25\right.$, and 0.0625$)$ for a sea bottom without a trench in a twolayer fluid. For this case it is observed that by increasing the distance of the barrier from the wall $b / h_{1}$, the value of the first peak frequency is reduced, similar to the trends observed in a single-layer fluid. This is may be due to the fact that by increasing the width of the device, the distance the upper and lower layer fluid particles must travel in a period of motion increases, which leads to a decrease in the value of $K h_{1}$ at which resonance occurs.

Fig. 2(b) shows the efficiency versus $K h_{1}$ for an OWC device near an asymmetric trench for different depth ratio values $h / h_{1}(=0,05,0.10,0.20,0.30,0.40)$. It is observed that the efficiency increases slightly as the depth ratio $h / h_{1}$ increases. This pattern is more significant when the interface is nearer and above the immersed tip of the barrier. This may be because internal waves incident to the barrier produce a highly resonant motion, due to their shorter 


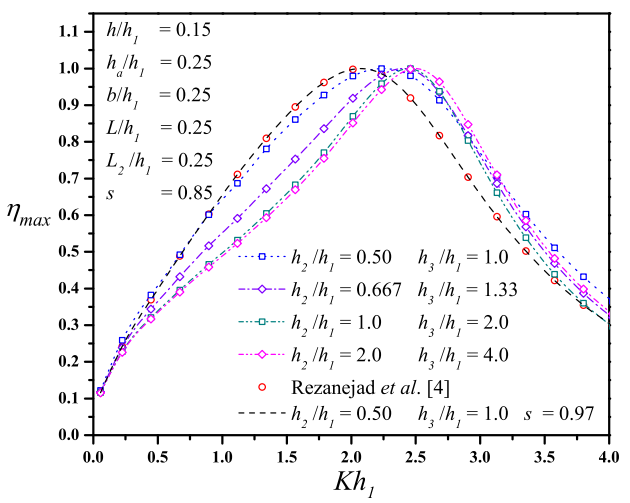

(a)

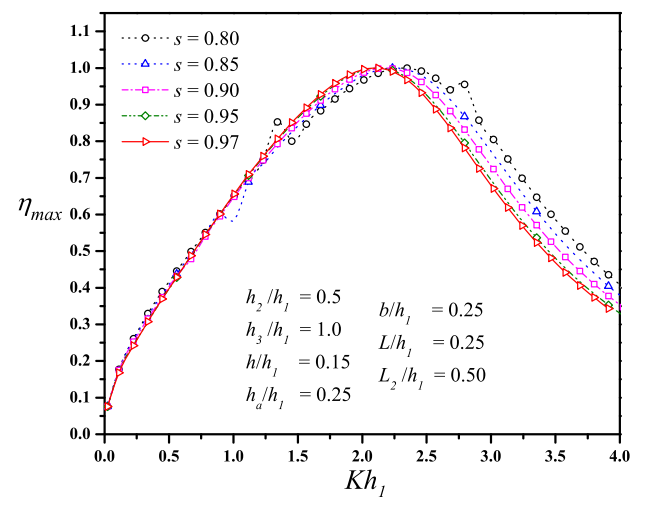

(b)

Figure 3: Analytical results for efficiency versus $K h_{1}$ for various locations of the trench and density ratios.

wavelengths with respect to the surface wavelength. Also, the interaction between the incident and reflected internal and surface waves affects the resonance frequency, which can lead to increased efficiency of the OWC.

Fig. 2(c) shows the results for the efficiency $\eta_{\max }$ versus $K h_{1}$ for various barrier to trench spacings $\left(L / h_{1}=0.75,1.00\right.$, and 1.25) in a two-layer fluid. It is observed that, for a symmetrical trench, the efficiency increases as the distance between the submarine trench and the water column barrier increases. This is due to the fact that the influence of the trench on the OWC performance is less significant when the distance between them grows, showing a similar pattern to that presented for a flat-bottom bathymetry.

In Fig. 3(a), the efficiency versus $K h_{1}$ is depicted for various locations of the trench. For values of less than 2.3 for the non-dimensional frequency, the trench has a decreasing effect on the efficiency compared with the case of a stepped bottom topography, $h_{2} / h_{1}=0.50$ and $h_{3} / h_{1}=1.0$, suggesting that the water depth in region 3 plays an important role, since the waves are reflected back, leading to a decrease in the capture of wave energy by the OWC device [10].

Fig. 3(b) plots efficiency versus $K h_{1}$ for an asymmetric trench for various density ratios $s(=0.80,0.85,0.90,0.95,0.97)$. The values of 0.95 and 0.97 correspond to common density ratios of a two-layer fluid consisting of freshwater and saltwater. Fig. 3(b) shows that the peak frequency moves slightly to the right-hand side and the frequency bandwidth slightly increases when the density ratio $s$ decreases.

\section{CONCLUSIONS}

The problem of an asymmetric submarine trench on the efficiency of an Oscillating Water Column device in a two-layer fluid was analyzed by employing the eigenfunction expansion method. The analytical results were compared with limiting cases obtained by Evans and Porter [3] and Rezanejad et al. [4] for the cases of horizontal and stepped bottom bathymetries, respectively, on the efficiency of an OWC device in a single-layer fluid and very good agreement was achieved. Numerical estimates for the efficiency were then obtained for various values of the physical parameters involved in the problem. In addition to the structural properties, it is observed that the interface position and the fluid density ratio affect the efficiency of an OWC device in a two-layer fluid. The depth of the submarine trench and 
the distance of the trench from the water column barrier also affect the OWC performance. In particular, for a symmetrical trench, the efficiency increases as the distance of the submarine trench from the water column barrier increases. Finally, it is hoped that the results from this study may provide valuable information for the clean and efficient harnessing of marine renewable energy.

\section{ACKNOWLEDGEMENTS}

The present research has been developed under the framework of CEMIE-Océano (Mexican Centre for Innovation in Ocean Energy). Project 249795 financed by CONACYT-SENERSustentabilidad Energética.

\section{REFERENCES}

[1] Kumar, P.S., Bhattacharjee, J. \& Sahoo, T., Scattering of surface and internal waves by rectangular dikes. Journal of Offshore Mechanics and Arctic Engineering, 129(4), pp. 306-317, 2007.

[2] Wehausen, J.V. \& Laitone, E.V., Surface waves. Encyclopedia of Physics, Vol. 9, Springer-Verlag: Berlin, pp. 446-778, 1960.

[3] Evans, D.V. \& Porter, R., Hydrodynamic characteristics of an oscillating water column device. Applied Ocean Research, 17(3), pp. 155-164, 1995.

[4] Rezanejad, K., Bhattacharjee, J. \& Guedes Soares, C., Stepped sea bottom effects on the efficiency of nearshore oscillating water column device. Ocean Engineering, 70, pp. 25-38, 2013.

[5] Kirby, J.T. \& Dalrymple, R.A., Propagation of obliquely incident water waves over a trench. Journal of Fluid Mechanics, 133, pp. 47-63, 1983.

[6] Das, D., Mandal, B.N. \& Chakrabarti, A., Energy identities in water wave theory for free-surface boundary condition with higher-order derivatives. Fluid Dynamics Research, 40(4), pp. 253-272, 2008.

[7] Panda, S. \& Martha, S.C., Oblique wave scattering by undulating porous bottom in a two-layer fluid: Fourier transform approach. Geophysical and Astrophysical Fluid Dynamics, 108(6), pp. 587-614, 2014.

[8] Evans, D.V., Wave-power absorption by systems of oscillating surface pressure distributions. Journal of Fluid Mechanics, 114, pp. 481-499, 1982.

[9] Smith, C.M., Some problems in linear water waves. PhD Thesis, University of Bristol, UK, 1983.

[10] Anvesh, V., Karmakar, D. \& Guedes Soares, C., Performance of oscillating water column wave energy converters integrated in breakwaters. Progress in Renewable Energies Offshore, ed. C. Guedes Soares, Taylor and Francis Group: London, pp. 295302, 2016. 